\title{
Acknowledgments
}

THIS PROJECT BEGAN as a panel organized for the $200 \mathrm{I}$ meeting of the American Anthropological Association, entitled "Cultural Intimacy and Mass Mediation: Articulating New Grounds for Ethnography between Public and Private Spheres." When I first invited colleagues to participate in the panel, I knew most of them only through their published work. My previous labors as an editor were "community-based," relying on long acquaintance and proximity, around which ideas developed. I have greatly enjoyed, this time around, building relationships on the topical affinities and theoretical resonances one finds in books. To the contributors I already considered friends, and to those who (often through the medium of email) have become close and reliable allies, I can happily point to yet another proof of the formative links between intimacy and mass mediation. Most of the panel's participants have carried their ideas through to the pages of this book, and those who joined later helped us amplify and expand the volume's central themes. I thank all the contributors for their enduring interest in the project and for the pleasure (a rare and not strictly editorial one) of reading their manuscripts over and over again, a task that has equipped me to think and argue differently and, in the process, to alter some old intellectual habits.

The essays "on display" in this volume have their own "off stage" areas of production. They have benefited greatly from the attention of many readers, not all of whom I can acknowledge personally, so I second the thanks offered elsewhere by individual contributors. Kay Warren, who read several of the papers in early drafts, encouraged me with her strong enthusiasm for the project, and Michael Herzfeld, whose ideas figure centrally in the volume, offered a judicious (and transformative) blend of criticism and support. It is safe to say the volume would never have materialized without his efforts, or his inspiration. The participants in "Cultural Intimacy and Mass Mediation," a seminar I taught at the University of Michigan in 2002, were a rich source of insight as well; many of my ideas made complete sense to me only after they had been tossed around by these sometimes skeptical, always creative students. 


\section{x Acknowledgments}

Two anonymous reviewers at Stanford University Press gave us fascinating commentary and lobbied for changes in ways that suggested a true appreciation for the project. Several essays changed radically (and for the better) in response to these readers. Our editors at the press, Pat Katayama and Carmen Borbón-Wu, brought the manuscript through its many stages of production, deftly and efficiently. Finally, I would like to thank my colleagues at the Center for Advanced Study in the Behavioral Sciences, especially members of the Identity and Difference reading group, whose discussions of their own work and mine supplied the perfect backdrop against which to write and edit this book. My year at CASBS (2002-2003) was generously supported by the Andrew W. Mellon Foundation (Grant \# 29800639). 